\title{
Short Range Order and Atomlc Charge Distribution In Amorphous Silicon
}

\author{
S. Kugler \\ Quantum Theory Group, Institute of Physics, Technical University of Budapest, \\ $\mathrm{H}-1521$ Budapest, Hungary
}

Keywords: Hydrogenated Amorphous Silicon, Structure, Short Range Order, Atomic Charge, Charge Fluctuation

\begin{abstract}
Our studies on the determination of the charge accumulation in finite aperiodic clusters of amorphous pure and hydrogenated silicon by means of a semiempirical molecular orbital method is summarized. An empirical formula depending on angle distortions is presented for calculating their net atomic charges. Applying the formula the charge fluctuations is calculated which are comparable to the experimental data.
\end{abstract}

\section{Introduction}

By symmetry the atoms in crystalline silicon has no charge at their equilibrium positions but in amorphous case they carry charges due to the geometric distortions [1-10]. These charges have never been directly measured though they play an important role in the determination of some experimental properties, like high-resolution core-level spectra or infrared absorptivity. Charge fluctuations in hydrogenated amorphous silicon $[2,10]$ and light-induced phenomena $[11,12]$ received much attention in the literature. It has been suggested that the Si-Si bond adjacent to the $\mathrm{Si}-\mathrm{H}$ bond becomes weaker than others and thus may be broken by prolonged illumination to create dangling bonds [11]. This might be the basis of the Staebler-Wronski effect [13], a reversible increase in the density of paramagnetic defects in a-Si:H caused by exposure to light.

In spite of theoretical understanding of electronic states in crystalline systems, amorphous atructures cause tnore difficult probloms for investigntions. Becnuse of the nbsenco of translational periodicity the electronic states can not be described within the context of Bloch's theory. The quantum chemical cluster methods are useful tools to investigate such systems. In these types of finite cluster Hartree-Fock (HF) culculations there is a difficulty in separating electron properties of the bulk and the surface becnuse of the nppearance of spurious surface states. The application of hydrogen for saturation of dangling bonds is a possible way. However, putting $H$ atoms onto the surface of a silicon cluster results in a 0.1 electron charge transfer within the H-Si bonds from hydrogen to silicon which may lead to artifacts in the calculations [14]. Increasing the size of the clusters is a further possible way to eliminate the influence of this effect but using $a b$ initio methods two other problems appear. There is a serious limit because of the computer capacity and over 10 silicon atoms the convergence problems increase. These problems reduce if a suitable semiempirical quantum chemical method is used.

Based on Strictly Localized Molecular Orbitals (SLMO) type calculations we propose an empirical formula for the calculation of net atomic charges in a-Si and a-Si:H. These charges are in a linear relationship with bond angle distortions involving first and second neighbours. 


\section{Methods}

Recently a quantum chemical method has been elaborated for the determination of net atomic charges in tetrahedrally coordinated amorphous materials such as silicon (or carbon) containing hydrogen $[9,15,16]$. A cluster of bulk atoms is described by a wave function expanded in terms of strictly localized molecular orbitals. SLMO's are linear combinations of $s p^{3}$ atomic hybrids directed along the corresponding bond axis and subsequently orthogonalized on each atom. Hybrid coefficients in SLMO's are derived through solution of a coupled $2 \times 2$ secular equation which is based on the diagonalization of a valence electron effective Hamiltonian. Owing to geometry distortions charges will accumulate on atoms and their relative magnitude can be estimated reliably by our method since inductive effects are correctly accounted for and the CNDO parametrization, we used, yields reliable charge distributions. In order to eliminate the edge effects we put pseudoatoms at the boundary modelling the bulk. These have only one or two neighbors to which they are connected by a sigma bond. The corresponding SLMO is composed of the appropriately oriented hybrids, one of them centered at pseudoatoms the other at the neighbouring atom. To ensure electroneutrality, the effective core charge of pseudoatoms is put equal to 1 or 2 depending on the number of hybrid orbitals attached to it.

A test of this model has been done for a crystalline silicon cluster containing 35 ordinary and 36 pseudoatoms. The first neighbour distances in a-Si and were put equal to $2.35 \AA$. The calculation yielded a uniformly neutral charge distribution, less than $10^{-4}$ electron units charge accumulation has been observed on any atom. This indicates that our method is free from the spurious accumulation of surface charges.

As a second test $a b$ initio quantum chemical calculations have been carried out on small a- $\mathrm{Si}: \mathrm{H}$ clusters in order to determine the net atomic charges and to make a comparison to SLMO type results. The clusters having 4-11 ordinary silicon atoms have been constructed on the basis of a computer simulated model structure for hydrogenated amorphous silicon [17]. To model the bulk we put hydrogen on the boundary. We have used the MUNGAUSS $a b$ initio program [18] and a standard 3-21G basis set. A fair linear correlation has been obtained between $a b$ initio and SLMO results. The ab initio charges are greater than the results calculated by the other method $\left(q_{a b}\right.$ initio $\left.\approx 1.4 q_{S L M O}\right)$. On the base of this comparison, we feel that SLMO is a very convenient method for calculating the net atomic charges since, as is known, HF ab initio method (with standard 3-21G basis set) a little bit overestimates the net atomic charges.

\section{Finite cluster models}

In crystalline silicon the nearest-neighbour arrangement of atoms is perfectly tetrahedral as expected from $s p^{3}$ hybridization. In the amorphous form the short range order is retained, only limited bond-length and bond-angle deviations can be found.

Some experimental structural studies has been made on a-Si using electron diffraction [19] and X-ray absorption [20], but still the elastic neutron scattering technique provides the most fundamental information about the structure. Recently different measurements on pure and hydrogenated amorphous silicon using neutron diffraction methods [21-25] have been published.

Many computer generated models [17, 25-37] have been constructed in different ways in the last decade. In the Monte Carlo simulation different types of local potential or even semiempirical fragment self-consistent field technique [38] were used as assumptions to minimize the total energy. The other models containing some threefold and fivefold coordinated 
atoms have been obtained by molecular dynamics technique. The ESR measurements show less than $10^{19} \mathrm{~cm}^{-3}$ intrinsic defects which is a very small number comparing to the fourfold coordinated atoms. Reverse Monte Carlo simulation [39,40], a new method for diffraction data analysis and/or structural modeling of amorphous silicon has been used for generating three dimensional particle configuration which are consistent with the measured structure factor $[25,36]$.

Additional information about the local order of the amorphous silicon might be provided by analyzing embedded silicon fragments inside the different kinds of molecules. The environment of silicon atoms might be considered as a "white noise" which is supposed to be similar to an amorphous arrangement.

A systematic analysis of structural data has been carried out by means of Cambridge Structural Database (CSD) [41]. This database contains results of different diffraction studies. We have collected the experimentally determined structural data on molecules containing at least, one $\mathrm{Si}-\mathrm{Si}-\mathrm{Si}$ fragment. Searching on the CSD, 384 targets have been found. The $\mathrm{R}$ factor of these data showing the accuracy of the measurements has been checked. Some of them were greater than 0.1 . We cancelled such data, because the accuracy of these measurements were found to be not satisfactory. $346 \mathrm{Si}-\mathrm{Si}-\mathrm{Si}$ fragments remained after this process.

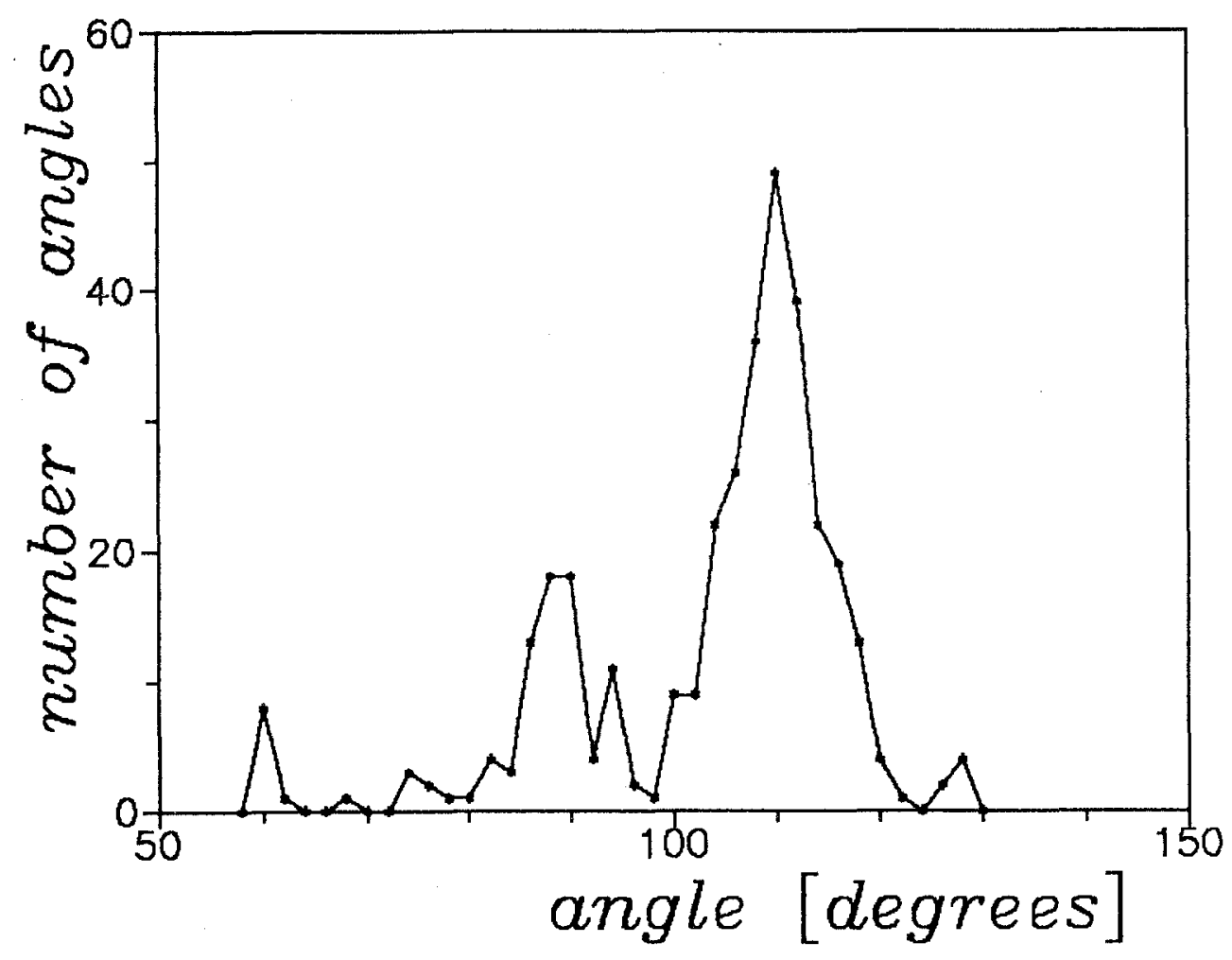

Figure 1. Bond angle distribution.

The hystogram of the bond angle distribution is shown in Fig. 1. The first conclusion of bond angle analysis is that the $\mathrm{Si}_{3}$ triangle is present among the fragments. The bond angles are about $60^{\circ}$ i.e. nearly equilateral triangle can be found. Most of the a-Si theoretical models don't contain such a part of the structure. There is a peak around $90^{\circ}$, too. It belongs to the nearly planar square arrangement. This type of four-membered ring is not so common comparing the models. The last conclusion of this hystogram is that greater than $130^{\circ} \mathrm{Si}-\mathrm{Si}-\mathrm{Si}$ bond angle has never been measured. 


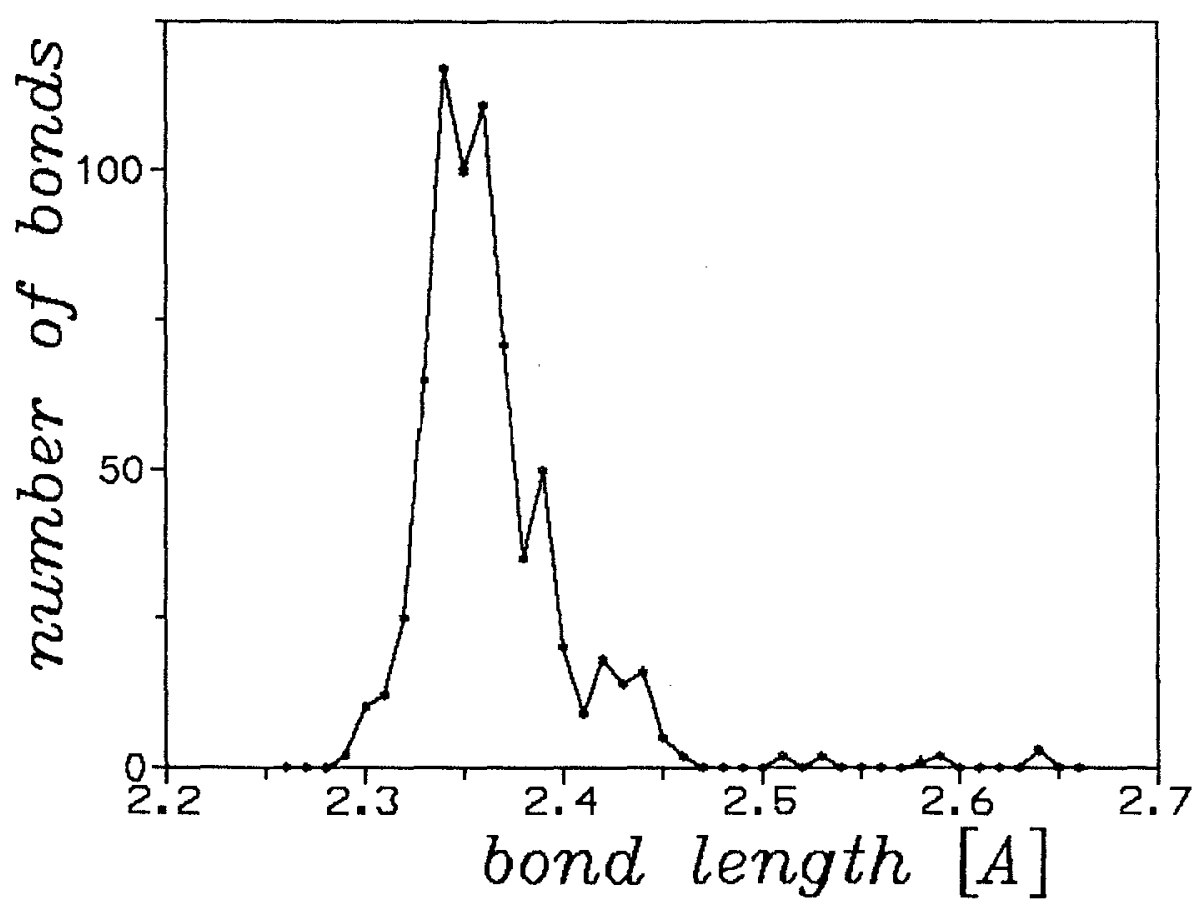

Figure 2. Bond length distribution.

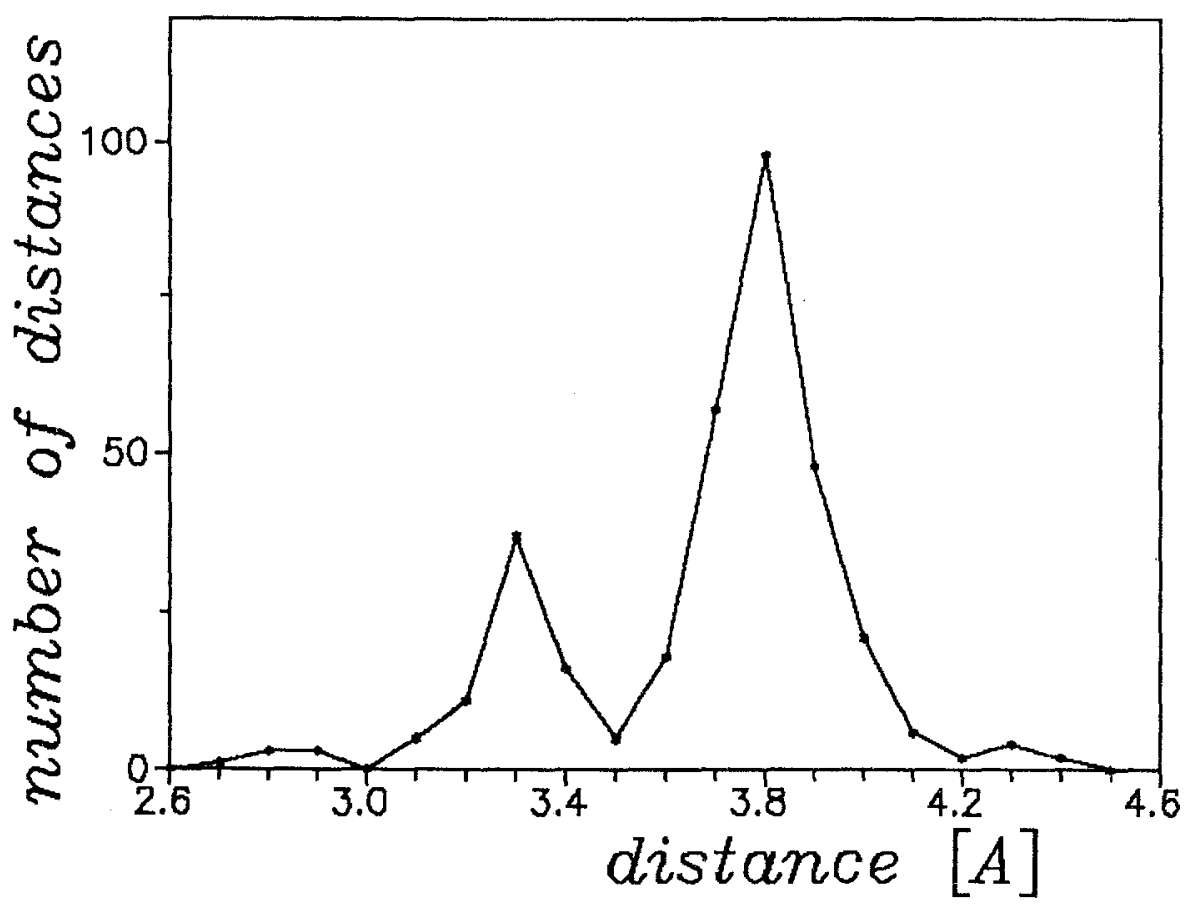

Figure 3. Second neighbour distribution.

The hystogram of bond length distribution is shown in Fig. 2. The bond length analysis shows that the lower limit is $2.29 \AA$. The upper limit is $2.65 \AA$ but there are very few bond lengths over $2.46 \AA$. The second neighbour distances are shown in Fig. 3. The peak around $3.3 \AA$ belongs to the nearly planar square arrangement. 
In our calculation we have used the continuous random network (CRN) model for the structure of a covalently bonded amorphous silicon. There are no coordination defects nor dangling bonds inside the model. The CRN model can be considered to be an idealized structure of bulk, tetrahedrally coordinated amorphous silicon. The finite clusters have been constructed on the basis of a CRN model for a-Si by Wooten et al. [26]. This, so-called Sillium model consists of a network of 216 silicon atoms interacting through the Keating local potential [42]. This Monte Carlo simulation seems to be the best CRN model at present since it has a periodic boundary condition and reproduces the experimental one-dimensional radial distribution function of tetrahedrally coordinated amorphous semiconductors excellently within the interval of $0-8 \AA[24]$.

\section{Amorphous silicon}

Atomic charge. Let us consider first the unhydrogenated amorphous silicon. Using the Wooten model we have built up finite a-Si clusters. Each of the model clusters consisted of at least 17 normal silicon atoms and more than 25 pseudoatoms located at the surface.

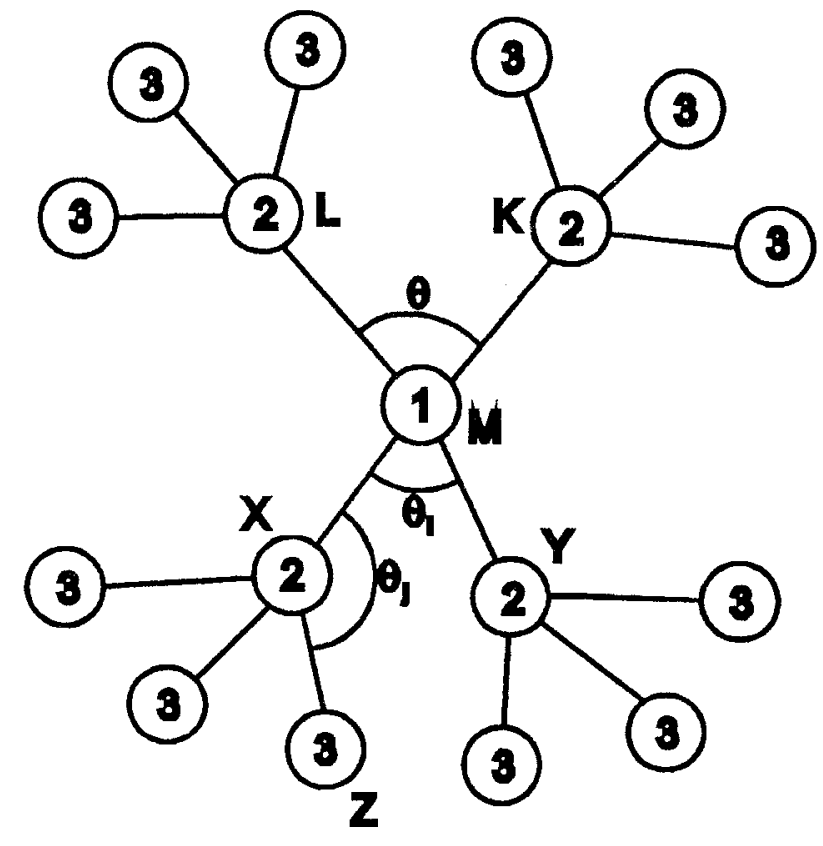

Figure 4. Cluster arrangement showing the central atom (1), first (2) and second (3) neighbour atoms

From the molecular orbital calculation we concluded that charge accumulation in tetrahedrally bonded semiconductors like a-Si is a three-atom effect, atomic net charges are generated by bond angle distortions. Let us consider an elementary triad of atoms, denoted by $K, L$ and $M$, forming two bonds KM and LM with a KML angle denoted by $\theta$ (Fig. 4). The net charge on the atoms of the triad depends linearly on the deviation of $\theta$ from the ideal tetrahedral value $\left(d \theta=\theta-109.47^{0}\right)$ : 


$$
\begin{gathered}
q_{M}^{e l}=2 A d \theta \\
q_{K}^{e l}=q_{L}^{e l}=-A d \theta,
\end{gathered}
$$

where $\mathrm{A}$ is a fitting parameter. The total atomic net charge on atom $\mathrm{M}$ is a sum of contributions originating from all combinations of the triads containing M. Since in the distorted tetrahedral model of Wooten each atom is at the center of 6 triads and at the end of 12 triads we have the following relation

$$
q_{M}=A\left(2 \sum_{i=1}^{6} d \theta_{i}-\sum_{j=1}^{12} d \theta_{j}\right)
$$

where $\theta_{i}=X M Y$ angle and $\theta_{j}=M X Z$ angle $(\mathrm{X}$ and $\mathrm{Y}$ are bonded to $\mathrm{M}, \mathrm{Z}$ to $\mathrm{X}$ or $\mathrm{Y}$, see Fig. 4).

We compared net charges, calculated by Eq.(2), to those obtained by our quantum chemical method. We constructed models, as described above, and only the five central silicon atoms were included in the comparison. As a whole, we calculated net charges for 79 different silicon atoms and obtained a fair linear correlation for Eq.(2) with $\mathrm{A}=-0.69$ millielectrons/degrees (cf. Fig. 5) [9].

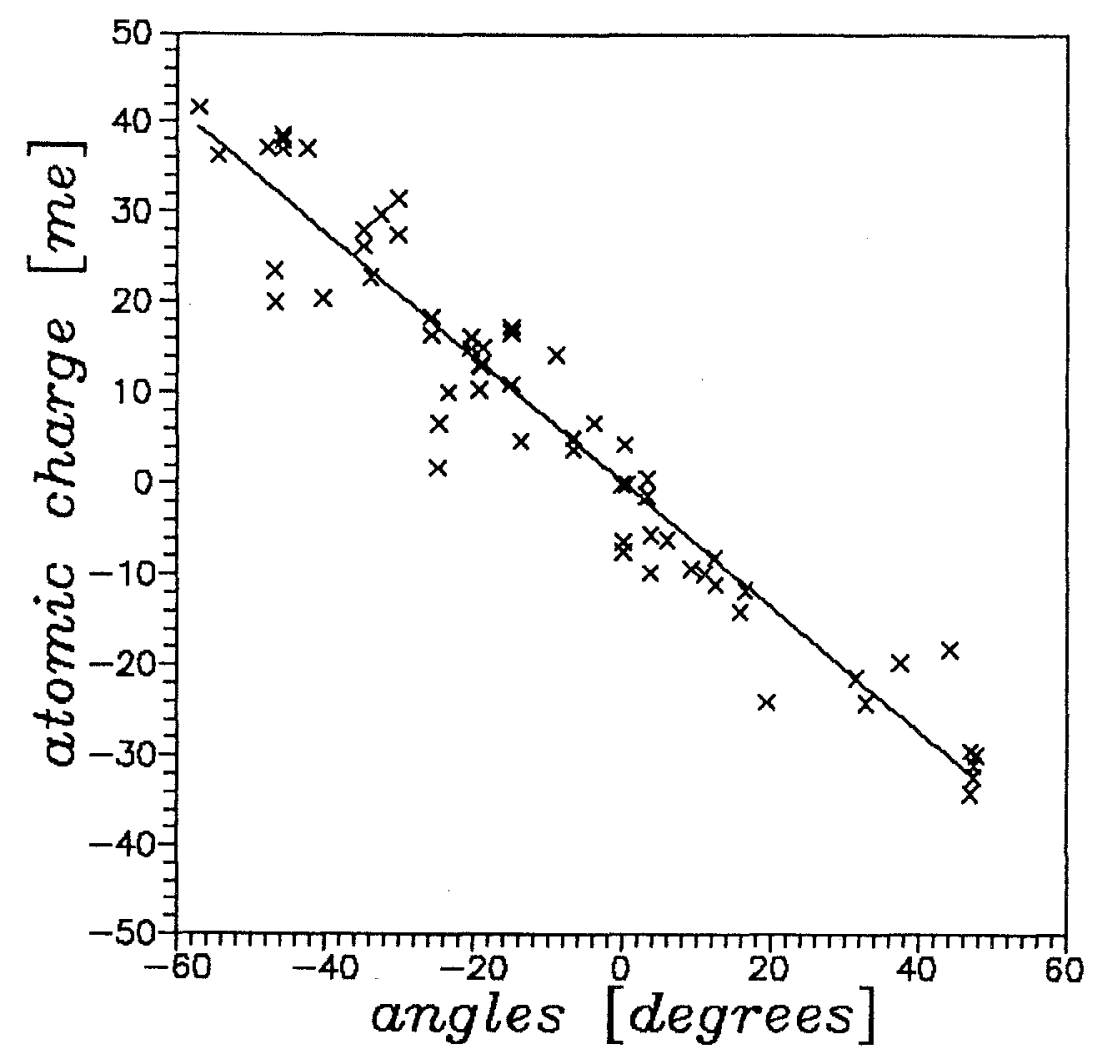

Figure 5. Comparison of atomic charges calculated by SLMO method (crosses) and by Eq.(2) (solid line). Angles (x axis) denotes the quality in parentheses in Eq. (2). 
Charge Fluctuations. To estimate charge fluctuations in a-Si we used the geometric model proposed by Wooten et al. [26]. Applying Eq.(2) for all 216 atoms of the model cluster we obtain $\mathrm{dq}_{S i}=0.021$ electron for the rms charge fluctuation. This is compared with values from other studies [1-5,8-9] in Table I. As it is seen, our estimate is considerably smaller

than those from experimental studies.

\begin{tabular}{cccc}
\hline No. & Authors & Type & $\mathrm{dq}_{S i}[\mathrm{me}]$ \\
\hline 1. & Guttman et al.[1] & theor. & 200 \\
2. & Ley et al.[2]. & exp. & 110 \\
3. & Klug, Whalley[3] & exp. & $180-320$ \\
4. & Kramer et al.[4] & theor. & 35 \\
5. & Bose et al.[8] & exp. & 140 \\
6. & Brey et al.[5] & exp. & $\leq 30$ \\
7. & Kugler et al.[9] & theor. & 21 \\
\hline
\end{tabular}

Table 1. Charge fluctuations $\left(\mathrm{dq}_{S i}\right)$ from theoretical and experimental studies in a-Si as obtained by different authors.
We feel that our value is realistic since, as it is known, CNDO/2 net charges reproduce absolute values of experimental dipole moments within a reasonable error. In our semiempirical quantum chemical method we used the CNDO $/ 2$ parametrization. The discrepancy with the core-level spectroscopic estimate of Ley et al. [2] may be explained on the basis of intra-atomic charge transfer [5] while the infra-red spectroscopic estimation is subject to considerable simplifications as discussed by the authors themselves [3]. In a real a-Si system there are a few so-called dangling bonds. Their number is about $10^{15}-10^{19} / \mathrm{mol}$. These defects also increase the charge fluctuation in a-Si.

We have estimated rms charge fluctuations in diamond-like a-C using all 216 atoms of the scaled model mentioned above. In order to derive the fitting parameter $\mathrm{A}$ for tetrahedrally bonded amorphous carbon, a similar process has been carried out. A fair linear correlation was obtained with $A=--0.51$ millielectrons/degrees for diamondlike a-C [43]. Applying again Eq.(2) we obtain dqC $=0.015$ electron units.

The charge distribution curve for a-Si, as obtained by smoothing the calculated histograms with Gaussian line broadening that corresponds to a full width of 0.064 electrons at half maximum has characteristic maxima or shoulders [7]. Analyzing the distribution of rings, we have found that the number of fivefold rings belonging to the same atom determines the charge distribution to a given extent. The charge averages for atoms with $0,1,2,3,4$ and 5 fivefold rings are $-0.023,-0.016,-0.005,0.009,0.021$ and 0.035 electrons, respectively. These values show fair coincidence with maxima on the charge distribution curve. Applying the above Gaussian line broadening method we smoothed the histogram displaying the number of atoms with $0,1,2,3,4$ and 5 fivefold rings (10, 45, 67, 64, 26 and 4 , respectively). The fair correspondence between both curves call the attention to the primary importance of ring statistics in determining the charge distribution [7].

\section{Hydrogenated amorphous silicon}

In order to determine charge fluctuation in a- $\mathrm{Si}: \mathrm{H}$ the same CRN model have been used as a basis. SLMO calculations have been performed on some clusters cut out from the 216 atom CRN model and modelled the bulk by pseudosilicon atoms as in a-Si case, then replaced one or more of these pseudoatoms by hydrogen. We have found that the quantum chemically determined net charges can be approximated by the following empirical formula: 


$$
q_{M}=A . S+B
$$

$\mathrm{A}$ is a constant (-0.69 millielectrons/degrees), $\mathrm{S}$ is the combination of bond-angle distortions

$$
S=\left(2 \sum_{i=1}^{6} d \theta_{i}-\sum_{j=1}^{12} d \theta_{j}\right)
$$

where $\theta_{i}=X M Y$ angle and $\theta_{j}=M X Z$ angle if the angle exists, elsewhere it is equal to zero ( $\mathrm{X}$ and $\mathrm{Y}$ are bonded to $\mathrm{M}, \mathrm{Z}$ to $\mathrm{X}$ or $\mathrm{Y}$ ).

The term B represents the inductive effect of hydrogen. SLMO calculations on crystal-like models show that this does not extend beyond the second neighbour of the hydrogen atom, i.e. putting $\mathrm{q}_{H}=-91, \mathrm{q}_{S i_{1}}=94, \mathrm{q}_{S i_{2}}=-1$ millielectrons we obtain a neutral cluster which is transferable from one model to the other. Thus, $\mathrm{B}$ is equal to $q_{S i_{1}}$ if $\mathrm{M}$ is adjacent to hydrogen, to $\mathrm{q}_{\mathrm{Si}_{2}}$ if it is the second neighbour and zero otherwise. Comparing Eq.(3) to SLMO calculations on some model clusters we obtain a fair agreement.

Now applying Eq.(3) to the 216 atom cluster of Wooten put by a varying number of hydrogen atoms upto 20 atomic percent we obtain the charge fluctuation as a function of hydrogen content $\left(\mathrm{a}-\mathrm{Si}_{(100-x)}: \mathrm{H}_{x}\right.$, cf. Fig. 6).

These values are compared to experiment investigation [2]. The measured charge transfer $\mathrm{dq}_{H}=0.15$ electrons from silicon atoms to $\mathrm{H}$, which is larger than expected from Pauling's electronegativy (0.02 electrons) but the measurement overestimates the charge transfer [9].

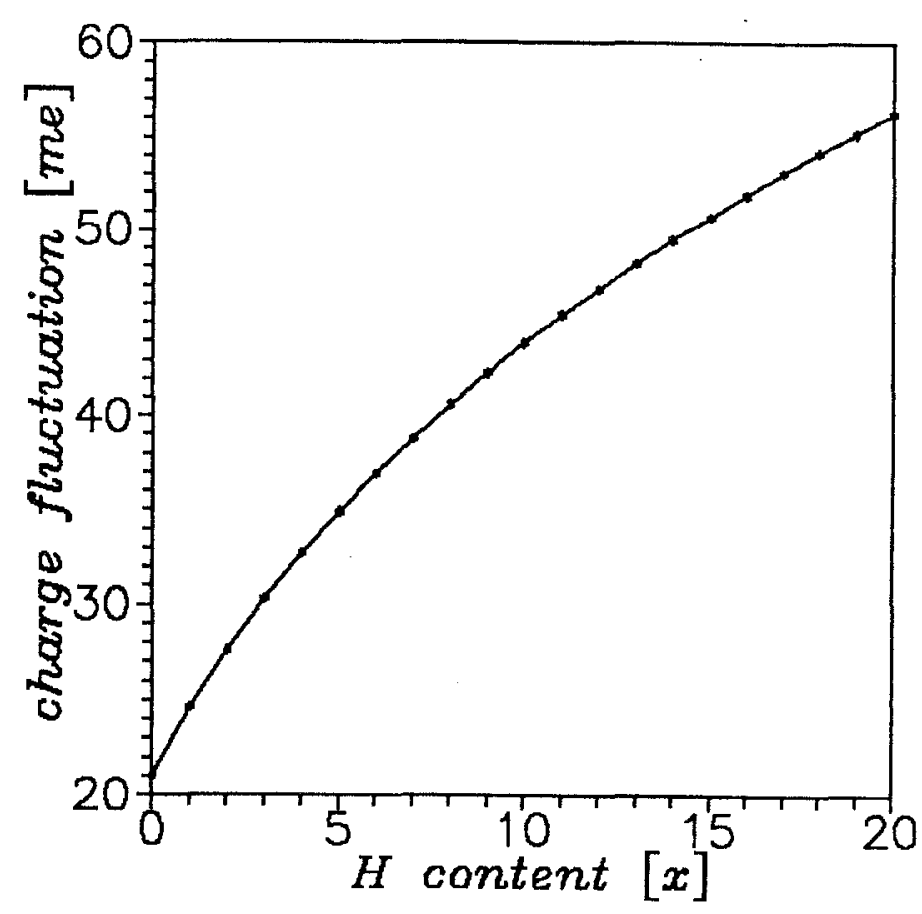
We think that our calculated value $\mathrm{dq}_{H}=0.091$ electron is more realistic.

Figure 6.

Bond lengths We made an attempt to check a statement on the weakening of $\mathrm{Si}-\mathrm{Si}$ bonds adjacent to the $\mathrm{Si}-\mathrm{H}$ bond by MNDO/PM3 molecular orbital calculations on small silanes $\left(\mathrm{Si}_{2} \mathrm{H}_{6}, \mathrm{Si}_{3} \mathrm{H}_{8}, \mathrm{Si}_{7} \mathrm{H}_{16}\right.$ and $\left.\mathrm{Si}_{8} \mathrm{H}_{18}\right)$. Considering geometry optimized structures of the above molecules we have found that the equilibrium bond length in the ground state depends on the number of hydrogen atoms attached to one or the other silicon forming the $\mathrm{Si}-\mathrm{Si}$ bond.

This is due to the accumulation of positive charges on silicon caused by the inductive effect of hydrogen. We found the following linear relationship between $\mathrm{Si}_{a}-\mathrm{Si}_{b}$ bond lengths and the sum of silicon net charges: 


$$
d_{S i_{a} S i_{b}}=0.038 \AA / e\left(q_{S i_{a}}+q_{S i_{b}}\right)+2.37 \AA
$$

where $d_{S i_{a} S i_{b}}$ is the distance between first silicon atoms and $q_{S i}$ is the atomic charge.

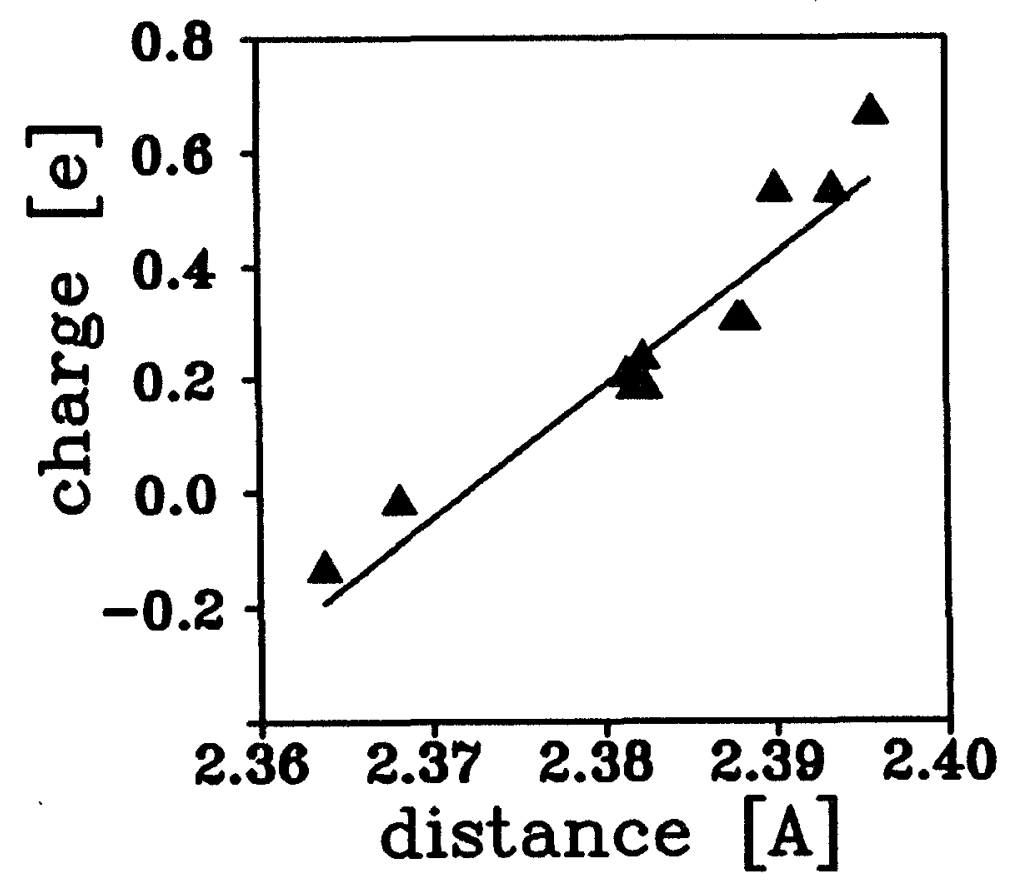

Figure 7

Atomic charges [in electrons] vs. bond distances [in Angstroms].

The relationship is graphically displayed in Figure 7. Though the above statements hold for the ground state there is some evidence that they can be generalized for the first excited state as well [10]. The lengthening is much more moderate but shows the same tendency both in the ground and first excited states.

As a consequence, we may state that atomic charge accumulation in pure and hydrogenated a-Si (and in diamondlike a-C) depend on the short range order. Primarily the charge distribution is determined by bond angle distortions.

\section{Acknowledgement}

The author acknowledge the helpful discussion with Prof. K. Morigaki (Tokyo), with Prof. G. Náray-Szabó and with P.R. Surján. This work was supported in part by the OTKA Grant No. T7283 and by MKM Grant No. 120/94.

\section{References}

[1] L. Guttman, W.Y. Ching and J. Rath, Phys. Rev. Lett. 44, 1513 (1980)

[2] L. Ley, J. Reichardt and R.L. Johnson, Phys. Rev. Lett. 49, 1664 (1982)

[3] D.D. Klug and E. Whalley, Phys. Rev. B 25, 5543 (1982) 
[4] B. Kramer, H. King and A. Mackinnon, J. Non-Cryst. Solids 59-60 73 (1983)

[5] L. Brey, C. Tejedor, and J.A. Verges, Phys. Rev. Lett. 52, 1840 (1984)

[6] K. Winer and M. Cardona, Solid State Comm. 60, 207 (1986)

[7] S. Kugler and G. Náray-Szabó, J. Non-Cryst. Solids 97 - 98, 503 (1987)

[8] S. K. Bose, K. Winer, and O. K. Andersen, Phys. Rev. B 37, 6262 (1988)

[9] S. Kugler, P. R. Surján and G. Náray-Szabó, Phys. Rev. B 37, 9060 (1988)

[10] S. Kugler and G. Náray-Szabó, J. Non-Cryst. Solids 137\&138 295 (1991)

[11] K. Morigaki, Jpn. J. Appl. Phys. 27163 (1988)

[12] M. Stutzmann, Charge-Induced Structural Relaxation in Amorphous Silicon, Festkörperprobleme, Vol. 28, (1988) ed. U. Rossler (Vieweg, Braunschweig) p.1.

[13] D.L. Staebler and C.R. Wronski, Appl. Phys. Lett. 31292 (1977)

[14] I. László, Int. J. Quant. Chem. 21, 813 (1982)

[15] P.R. Surján, G. Náray-Szabó and I.Mayer, Int. J. Quantum Chem. 22929 (1982)

[16] P.R. Surján, The Two-Electron Bond as a Molecular Building Block, in Theoretical Models of Chemical Bonding, Part 2. The Concept of the Chemical Bond, ed. by Z. B. Maksic, (Springer, Heidelberg 1989)

[17] N. Mousseau and L. J. Lewis, Phys. Rev. B 413702 (1990)

[18] M. R. Peterson and R. A. Poirier, Program MUNGAUSS, Department of Chemistry, University of Toronto, Ontario and Chemistry Department, Memorial University, Newfoundland, St. John's, Newfoundland, Canada A1b 3X7

[19] A. Barna, P.B. Barna, G. Radnóczi, L. Tóth, and P. Thomas, Phys. Stat. Sol.(a) 41, $81(1977)$

[20] R. J. Ternkins, W. Paul, and G. A. N. Connell, Advances in Physics 22, 581 (1973)

[21] R. Bellissent, J. Non-Cryst. Solids 97\&98, 329 (1987)

[22] A. Menelle, J. Non-Cryst. Solids 97\&98, 337 (1987)

[23] R. Bellissent, A. Menelle, W.S. Howells, A.C. Wright, T.M. Brunier, R.N. Sinclair and F. Jansen, Physica B 156\&157, 217 (1989)

[24] S. Kugler, G. Molnár, G. Petö, E. Zsoldos, L. Rosta, A. Menelle and R. Bellissent, Phys. Rev. B. 40, 8030 (1989)

[25] S. Kugler, L. Pusztai, L. Rosta, P. Chieux and R. Bellissent, Phys. Rev. B 487685 (1993)

[26] F. Wooten, K. Winter and D. Weaire, Phys. Rev. Lett. 541392 (1985)

[27] T. Uda, Solid State Commun. 64, 837 (1987)

[28] M. D. Kluge, J. R. Ray, and A. Rahman, Phys. Rev. B 36, 4234 (1987)

[29] R. Biswas, G. S. Grest, and C. M. Soukoulis, Phys. Rev. B 36, 7437 (1987)

[30] R. Car and M. Parrinello, Phys. Rev. Lett. 60, 204 (1988)

[31] W. D. Luedtke and U. Landman, Phys. Rev. B 37, 4656 (1988)

[32] J. Tersoff, Phys. Rev. B 38, 9902 (1988)

[33] W. D. Luedtke and U. Landman, Phys. Rev. B 40, 1164 (1989)

[34] I. Stich, R. Car and M. Parrinello, Phys. Rev. B 44, 11092 (1991)

[35] J. M. Holender and G. J. Morgan, J. Phys., Condens. Matter 3, 1947 (1991)

[36] S. Kugler and L. Pusztai, Non-Cryst. Solids 164-166 147 (1993)

[37] G. Tóth and G. Náray-Szabó, J. Chem. Phys. 1003742 (1994)

[38] G. G. Ferenczy, J. L. Rivail, P. R. Surján and Náray-Szabó, J. Comput. Chem. 13830 (1992)

[39] R.L. McGreevy and L. Pusztai, Molec. Sim. 1, 369 (1988)

[40] O. Gereben and L. Pusztai, Phys. Rev. B. 50, 14136 (1994)

[41] F. H. Allen, O. Kennard and R. Taylor, Acc. Chem. Res. 16, 146 (1983)

[42] P. N. Keating, Phys. Rev. 1456371966.

[43] S. Kugler and G. Náray-Szabó, Jpn. J. Appl. Phys, Lett. 3020 (1991). 